\title{
NLRP3/ASC-mediated alveolar macrophage pyroptosis enhances HMGB1 secretion in acute lung injury induced by cardiopulmonary bypass
}

\author{
Lei Hou $^{1} \cdot$ Zhongwei Yang $^{1} \cdot$ Zhankui Wang $^{1} \cdot$ Xiao Zhang $^{1} \cdot$ Yanhua Zhao ${ }^{1} \cdot$ Hao Yang $^{1} \cdot$ Beijie Zheng $^{1} \cdot$

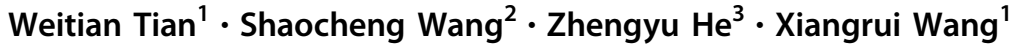

Received: 12 December 2017 / Revised: 16 March 2018 / Accepted: 2 April 2018 / Published online: 8 June 2018

(c) United States \& Canadian Academy of Pathology 2018

\begin{abstract}
Our previous study showed that high levels of HMGB1 existed in rats following cardiopulmonary bypass (CPB)-induced acute lung injury (ALI) and neutralization of high-mobility group box 1(HMGB1) reduced CPB-induced ALI. However, the mechanism by which CPB increases HMGB1 secretion is unclear. Recent studies have shown that inflammasome-mediated cell pyroptosis promotes HMGB1 secretion. This study aimed to investigate the relationship between inflammasomemediated pyroptosis and HMGB1 in CPB-related ALI. We employed oxygen-glucose deprivation (OGD)-induced alveolar macrophage (AM) NR8383 pyroptosis to measure HMGB1 secretion. We found that OGD significantly increased the levels of caspase- 1 cleaved p10, IL-1 $\beta$ and ASC expression, caspase- 1 activity and the frequency of pyroptotic AM, and promoted the cytoplasm transportation and secretion of HMGB1, which were significantly mitigated by ASC silencing or pretreatment with glyburide (a Nlrp3 inhibitor) in AM. CPB also increased the expression levels of Nlrp3, ASC, caspase-1 P10, and IL-1 $\beta$, and the percentages of AM pyroptosis in the lungs of experimental rats accompanied by increased levels of serum and bronchoalveolar lavage fluid (BALF) HMGB1. Treatment with glyburide significantly mitigated the CPB-increased ASC, caspase-1 p10 and IL-1 $\beta$ expression, and the percentages of AM pyroptosis in the lungs, as well as the levels of HMGB1 in serum and BALF in rats. Therefore, our data indicated that the Nlrp3/ASC-mediated AM pyroptosis increased HMGB1 secretion in ALI induced by CPB. These findings may provide a therapeutic strategy to reduce lung injury and inflammatory responses during $\mathrm{CPB}$.
\end{abstract}

These authors contributed equally: Lei Hou, Zhongwei Yang.

Electronic supplementary material The online version of this article (https://doi.org/10.1038/s41374-018-0073-0) contains supplementary material, which is available to authorized users.

Zhengyu He

zhengyuheshsmu@163.com

$\triangle$ Xiangrui Wang

xiangruiwang2016@163.com

1 Department of Anesthesiology, Ren Ji Hospital, School of Medicine, Shanghai Jiao Tong University, 200127

Shanghai, China

2 Department of Anesthesiology, Shanghai Tenth People's Hospital, School of Medicine, Shanghai Tong Ji University, 200072 Shanghai, China

3 Department of Critical Care Medicine, Ren Ji Hospital, School of Medicine, Shanghai Jiao Tong University, 200127

Shanghai, China

\section{Introduction}

Cardiopulmonary bypass (CPB) can cause acute lung injury (ALI), which remains a serious complication [1]. During the process of $\mathrm{CPB}$, pulmonary ischemia/perfusion (I/R) can induce oxidative stress and inflammation, leading to lung damages [2]. Hence, control of inflammation will be of significance in management of patients with CPB.

High-mobility group box 1 (HMGB1) is a ubiquitous nuclear protein that can be released by activated alveolar macrophage (AM) during the process of pulmonary $I / R$ [3]. The secreted HMGB1 can induce acute injury and inflammation [4]. Our previous study has found that HMGB1 is crucial for the development of ALI following hepatic I/R and CPB [5, 6]. HMGB1 can feedback enhance the activity of AM through toll-like receptor 4 (TLR4) to produce high levels of pro-inflammatory cytokines, contributing to the progression of ALI [7]. 
Our preliminary study indicated that during the process of $\mathrm{CPB}$, pulmonary I/R promoted abnormal high levels of HMGB1 secretion from AM, and neutralization of HMGB1 by specific antibodies alleviated lung injury during $\mathrm{CPB}$. Accordingly, inhibition of HMGB1 secretion during CPB may be valuable in reducing lung inflammation and alleviating lung injury. However, the mechanisms underlying abnormal HMGB1 secretion from AM during CPB remain unclear.

Pyroptosis is a novel form of programmed cell death, with unique morphological and biochemical features that are different from necrosis and apoptosis [8]. Pyroptosis is commonly observed in infected macrophages and dendritic cells, depending on the caspase-1 activation [9]. It is well known that during the process of pyroptosis, inflammasomes can activate and cleave caspase- 1 into casapse- 1 p10 and caspase-1 p20 to form membrane pores (1.1-2.4 nm), swelling, DNA fragmentation, and eventually osmotic lysis of macrophages, leading to effusion of inflammatory cytokines such as IL-1 $\beta$ and IL-18 [10]. Nlrp3 inflammasome is one of the four inflammasomes (Nlrp1, Nlrp3, Ipaf, and AIM2) [10, 11], which can directly interact with the adaptor ASC to activate caspase-1, leading to cell pyroptosis [10, 12]. Furthermore, Nlrp3 can also interact with caspase-8 or caspase-11 through a non-canonical inflammasome pathway to promote IL- $1 \beta$ and IL-18 maturation particularly during bacterial infection [13, 14]. In addition, intracellular lipopolysaccharides (LPS) from bacterial infection can activate caspase-11 and cleave gasdermin D, leading to NLRP3dependent caspase-1 activation and pyroptosis [15]. Moreover, Nlrp3 regulates the development of dendritic cells during chronic bacterial infection in a non-canonical inflammasome and ASC-independent manner [16], and nuclear Nlrp3 can interact with the transcription factors to promote cytokine production in Th2 cells [17]. Previous studies have shown that Nlrp3 is crucial for $\mathrm{I} / \mathrm{R}$-induced brain injury [18], and Nlrp3 silencing ameliorates the I/Rinduced myocardial infarct [19]. Nlrp3 can stimulate high levels of IL-1 $\beta$ and IL-18 production during the process of I/R-induced renal injury [20]. A recent study has shown that the ASC-mediated caspase-1 activation can promote abnormal secretion of HMGB1 during the process of hepatic I/R injury [21]. Subsequently, HMGB1 can feedback enhance macrophage cell pyroptosis through ASCinduced caspase-1 activation [22]. Accordingly, we hypothesize that Nlrp3/ASC-induced AM pyroptosis may mediate HMGB1 abnormal secretion during the process of ALI induced by $\mathrm{CPB}$.

In this study, we employed a rat model of CPB and in vitro cell model of oxygen and glucose deprivation to examine whether the Nlrp3/ASC-induced AM pyroptosis and HMGB1 secretion leads to ALI.

\section{Materials and methods}

\section{Experimental animals}

The experimental protocols were approved by the Animal Care and Use Committee of School of Medicine, Shanghai Jiaotong University (Shanghai, China). All animals received human care, according to the guidelines for animal care published by the National Institutes of Health of United States. Male Sprague-Dawley (SD) rats at 12 weeks of age and weighing $400-450 \mathrm{~g}$ were purchased from Shanghai Laboratory Animal Company (SLAC, Shanghai, China). The rats were housed in a specific pathogen-free facility with a cycle of 12-h light/dark and free access to food and water.

\section{Surgical procedures}

A rat model of CPB was established, as described previously with some modifications [23]. Briefly, individual rats were injected intraperitoneally with $40 \mathrm{mg} / \mathrm{kg}$ pentobarbital and inserted with a 14-G cannula into the trachea, followed by mechanically ventilated with $8 \mathrm{ml} / \mathrm{kg}$ tidal volume, the Peak End Expiratory Pressure (PEEP) of $5 \mathrm{~cm} \mathrm{H} \mathrm{H}_{2} \mathrm{O}$, respiration rate of 60 cycles per minute, and $30 \%$ oxygen (TOPO ventilator, USA). The rat was administrated systemically with heparin $(250 \mathrm{IU} / \mathrm{Kg})$ and its right femoral artery was cannulated with a 22-G catheter (Sinorgmed, Shandong, China) to monitor the mean arterial pressure (MAP) with a blood pressure instrument (HEWLETT PACKARD, HP, Germany) and to examine the arterial blood gas using the instrument (GEM3000, GE Health, USA). The tail artery was cannulated with a 22$G$ catheter for arterial infusion during the CPB circuit. The right external jugular vein was inserted with a $14-\mathrm{G}$ catheter to drain blood by gravity to the venous reservoir. The venous reservoir was connected with a tubing roller pump (Masterflex L/S, USA) and rat membrane oxygenator through special pipe (Xijing Medical Instrument, China). The circuit was connected with the infusion line. The roller pump was used to infuse the oxygenated venous blood to the rat body at a rate of $120-140 \mathrm{ml} / \mathrm{kg} / \mathrm{min}$ for $1 \mathrm{~h}$. After $1 \mathrm{~h}$, the cannulas were removed and the remaining blood in the pipe was gradually transferred to the rat. The incisions were sutured. The sham control rats were anesthetized, ventilated, and cannulated, but did not receive CPB.

In addition, some control and experimental rats were injected intraperitoneally $1 \mathrm{~h}$ before the sham or CPB with $50 \mathrm{mg} / \mathrm{kg}$ glyburide (Sigma-Aldrich), which is an antidiabetic drug and inhibitor of the NLRP3 inflammasome [24]. 
After the CPB surgery, the rats were observed for their recovery in the observation box. Blood samples were collected from the right atrium of individual rats at $6 \mathrm{~h}$ post $\mathrm{CPB}$, and the serum samples were prepared by centrifugation. Individual rats were anesthetized and subjected to bronchoalveloar lavage. Briefly, the rat was inserted with one 14-G catheter into the trachea and lavaged the both lungs with $10 \mathrm{ml}$ of PBS. The collected BALF was filtered through a $70-\mu \mathrm{m}$ BD falcon cell strainers and centrifuged at $700 \times g$ for $10 \mathrm{~min}$. After lysis of red blood cells, the remaining cells were characterized by anti-CD68 staining and immunofluorescence. The cells contained $99 \%$ of AM [25]. The rats were sacrificed and their partial lung tissues were fixed in $4 \%$ paraformaldehyde for $24 \mathrm{~h}$, gradient dehydrated in $20-30 \%$ sucrose, and embedded in OCT. Some lung tissues from individual rats were frozen in liquid nitrogen and homogenized. The tissue lysates were used for measurement of caspase-1 activity, total RNA extraction, and Western blot assays. The remaining lung tissues from the individual rats were fixed in $10 \%$ neutralized formalin overnight and paraffinembedded.

\section{AM and establishment of a cellular model of oxygen-glucose deprivation}

Rat AM NR8383 cells were purchased from the Cell Bank of the Chinese Academy of Sciences (Shanghai, China). The cells were regularly cultured in Ham's F-12k medium (1.2 g/L glucose, Invitrogen, USA), containing $15 \%$ fetal bovine serum (FBS, Hyclone, USA), at $37^{\circ} \mathrm{C}$ in $95 \%$ air and $5 \% \mathrm{CO}_{2}$. To expose to OGD, the cells were cultured in glucose-free Ham's F-12k culture medium in an oxygenfree $\mathrm{N}_{2} / \mathrm{CO}_{2}(95 / 5 \%)$ atmosphere for $6 \mathrm{~h}$, and changed to the regular condition for $6 \mathrm{~h}$.

In some experiments, the control and experimental groups of cells were pre-treated with $200 \mu \mathrm{M}$ glyburide [26] in glucose-free Ham's F-12k medium for 30 min and subjected to oxygen deprivation or regular culture. In addition, NR8383 cells were cultured at 50\% confluency and transfected with $50 \mathrm{nM}$ ASC-specific siRNA (5-GACAGCGGAUGAGUUUAAA-3) or scramble siRNA (scrbl siRNA), designed and synthesized by Genemeditech (Shanghai), using lipofectamine 2000 (Invitrogen) for $48 \mathrm{~h}$. The efficacy of ASC silencing was determined by qRT-PCR and Western blot. The cells were subjected to regular culture or OGD.

\section{Western blot}

Fresh lung tissues from individual rats were homogenized and lyzed in RIPA lysis buffer (Beyotime Biotechology, China). Different groups of AM cells were lyzed in
RIPA lysis buffer. Their cytoplasmic proteins were extracted using the NE-PER kit (Thermoscientific, USA). After quantification of protein concentrations, individual lysate samples $(30 \mu \mathrm{g} / \mathrm{lane})$ were separated by sodium dodecyl sulfate polyacrylamide gel electrophoresis (SDS-PAGE) on 10-15\% gels (Beyotime Biotechology) and transferred onto polyvinylidene difluoride (PVDF) membranes. The membranes were treated with $5 \%$ fat-free dry milk in TBST for $1 \mathrm{~h}$ and incubated with primary antibodies, including anti-Nlrp3 (1:1000 abcam), anti-ASC (1:200), anti-pro-caspase-1 (1:200), anti-capase-1 p10 (1:200 Santa Cruz Biotech, USA), and anti- $\beta$-actin $(1: 10,000$, CST) at $4{ }^{\circ} \mathrm{C}$ for $14 \mathrm{~h}$. After being washed, the bound antibodies were detected with horseradish peroxidase (HRP)-conjugated secondary anti-rabbit IgG (1:2000, Beyotime Biotechology) for $1 \mathrm{~h}$ at room temperature and visualized using the enhanced chemiluminescent reagent, followed by imaging using the Bio-Rad imaging system. The relative levels of the target protein to the control $\beta$-actin were quantified using the Image Pro Plus 5.1 software.

\section{Analysis of AM pyroptosis}

The collected AM cells from the BALF of individual rats were stained with Alexa Fluor 488-labeled anti-caspase-1 at $37^{\circ} \mathrm{C}$ for $1 \mathrm{~h}$ using the FAM-FLICA Caspase Assay Kit (ImmunoChemistry Technology, Bloomington, MN, USA), according to the manufacturer's instruction. After being washed, the cells were stained with propidium iodide (PI) for $5 \mathrm{~min}$ at room temperature, and the percentages of Caspase-1 + PI pyroptotic AM were analyzed by flow cytometry in Beckman Coulter CytoFlex instrument.

\section{Immunofluorescence staining}

The different groups of NR8383 cells were fixed with $4 \%$ paraformaldehyde, then were treated with $0.1 \%$ Triton $\mathrm{x}-100$, blocked with $10 \%$ BSA in PBS, and incubated with rabbit anti-HMGB1 (1:350) or rabbit antiASC (1:50) at $4{ }^{\circ} \mathrm{C}$ overnight. After being washed, the sections were incubated with $\mathrm{Cy} 3$-conjugated goat antirabbit IgG (1:500 Beyotime Biotechology) for $1 \mathrm{~h}$ at room temperature, followed by counterstaining with DAPI. The cells were imaged under a confocal microscope (Zeiss LSM710).

Similarly, the collected AM cells from the BALF of rats were fixed with $4 \%$ paraformaldehyde and treated with $0.1 \%$ Triton $\mathrm{x}-100$. After being washed, the cells were blocked with $10 \%$ BSA in PBS, and incubated with mouse anti-CD68 (1:100 Abcam) at $4{ }^{\circ} \mathrm{C}$ overnight. After being washed, the cells were incubated with $\mathrm{Cy} 3$-conjugated goat anti-mouse $\operatorname{IgG}(1: 1000$ Abcam $)$ for $1 \mathrm{~h}$ at room 
A
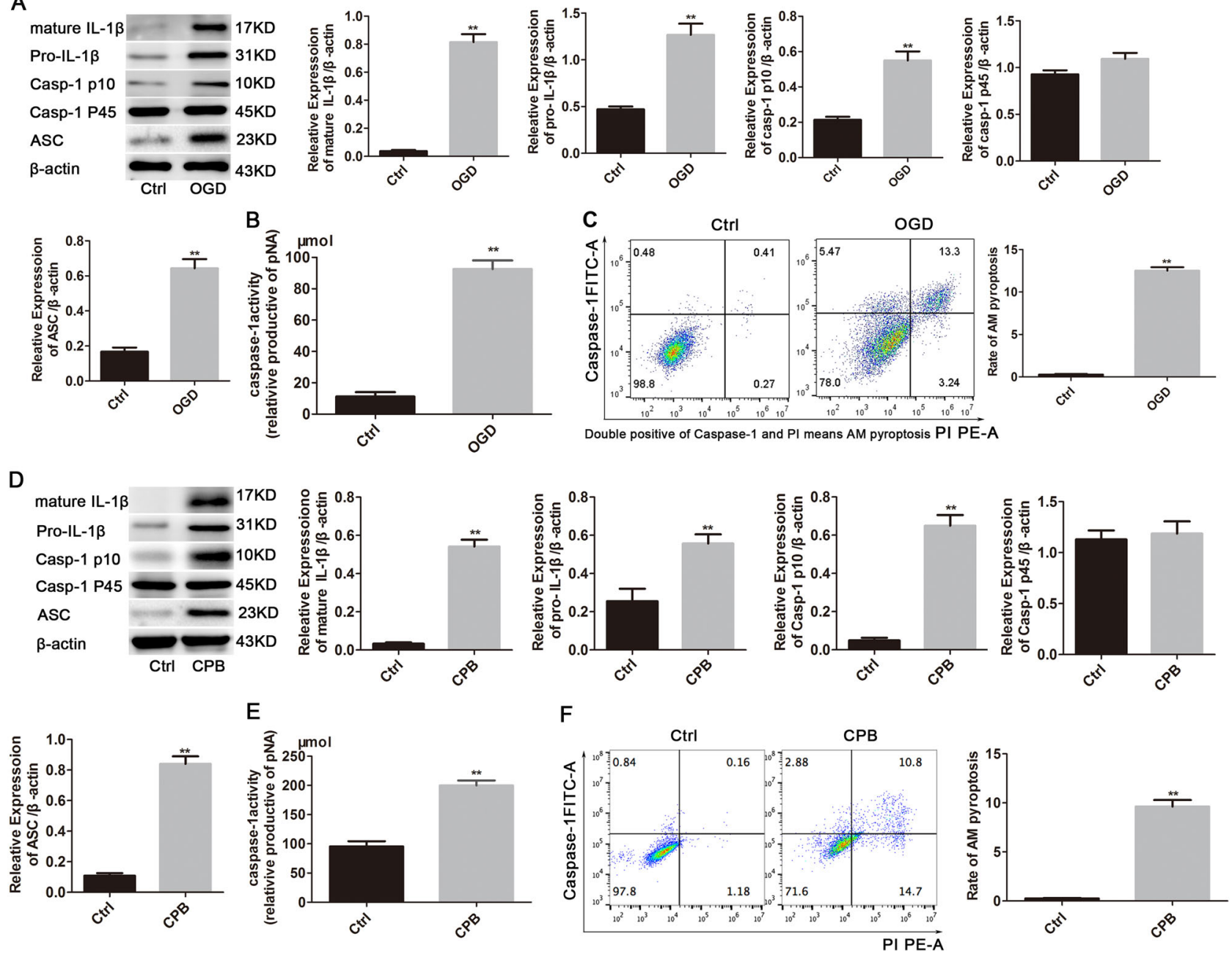

Fig. 1 Both OGD and CPB induce AM pyroptosis. Rat AM NR8383 cells were exposed to OGD or cultured in regular medium (control) for $6 \mathrm{~h}$ and cultured in regular medium for another $6 \mathrm{~h}$. In addition, rats were subjected to CPB or sham surgery and sacrificed at $6 \mathrm{~h}$ postsurgery. The relative levels of caspase- $1 \mathrm{p} 45$, p10, pro-IL- $1 \beta$, mature IL-1 $\beta$, ASC to the control $\beta$-actin expression in the different groups of $\mathrm{AM}$ and the lung tissues were determined by Western blot. The levels of caspase- 1 activity in AM and the lung were determined by the production of pNA, and the frequency of caspase-1 + PI pyroptotic

temperature and counterstained with DAPI. The cells were imaged under a Fluorescence microscope (Leica).

\section{Real-time PCR}

Total RNA was extracted from individual lung tissues and cell samples using the Trizol (Invitrogen), and each sample (1 $\mu \mathrm{g}$ ) of RNA was transcribed reversely into cDNA using the High Capacity cDNA Reverse Transcription Kit (Thermofisher). The relative levels of target gene mRNA transcripts to the control GAPDH were determined by quantitative RTPCR using the $\mathrm{SYBR}^{\circledR}$ Green Real-Time PCR Master Mixes (Thermofisher) and specific primers. The sequences of
AM were characterized by flow cytometry. a, $\mathbf{d}$ Western blot analysis of the pyroptosis-related protein expression in AM and the lung, respectively. b, e The levels of caspase-1 activity in AM and the lung, respectively. c, $\mathbf{f}$ The frequency of pyroptotic AM and BALF AM, respectively. Data are representative images or expressed as the mean \pm SEM of each group ( $n=3$ per group for in vitro assay; $n=6$ per group of rats) from three separate experiments. ${ }^{*} p<0.01$ vs. the control group

primers were sense 5'-CCCTCATGTTGCCTGTTCTT-3' and antisense $5^{\prime}$-ACCTCGGCAGAAGCTAGAG-3' for rat Nlrp3; and sense 5'-AGACCTCTATGCCAACACAGTGC$3^{\prime}$ and antisense 5'-GAGCCACCAATCCACACAGAGT-3' (antisense) for rat GAPDH. Amplification reactions were performed in duplicate at $95{ }^{\circ} \mathrm{C}$ for $30 \mathrm{~s}$, and subjected to 40 cycles of $95{ }^{\circ} \mathrm{C}$ for $3 \mathrm{~s}$ and $62{ }^{\circ} \mathrm{C}$ for $30 \mathrm{~s}$. The data were analyzed by the $2^{-\Delta \Delta \mathrm{CT}}$ method and expressed as fold change.

\section{ELISA}

The levels of HMGB1 and IL-1 $\beta$ in sera, BALF, and cell supernatants were assessed by ELISA using the specific 

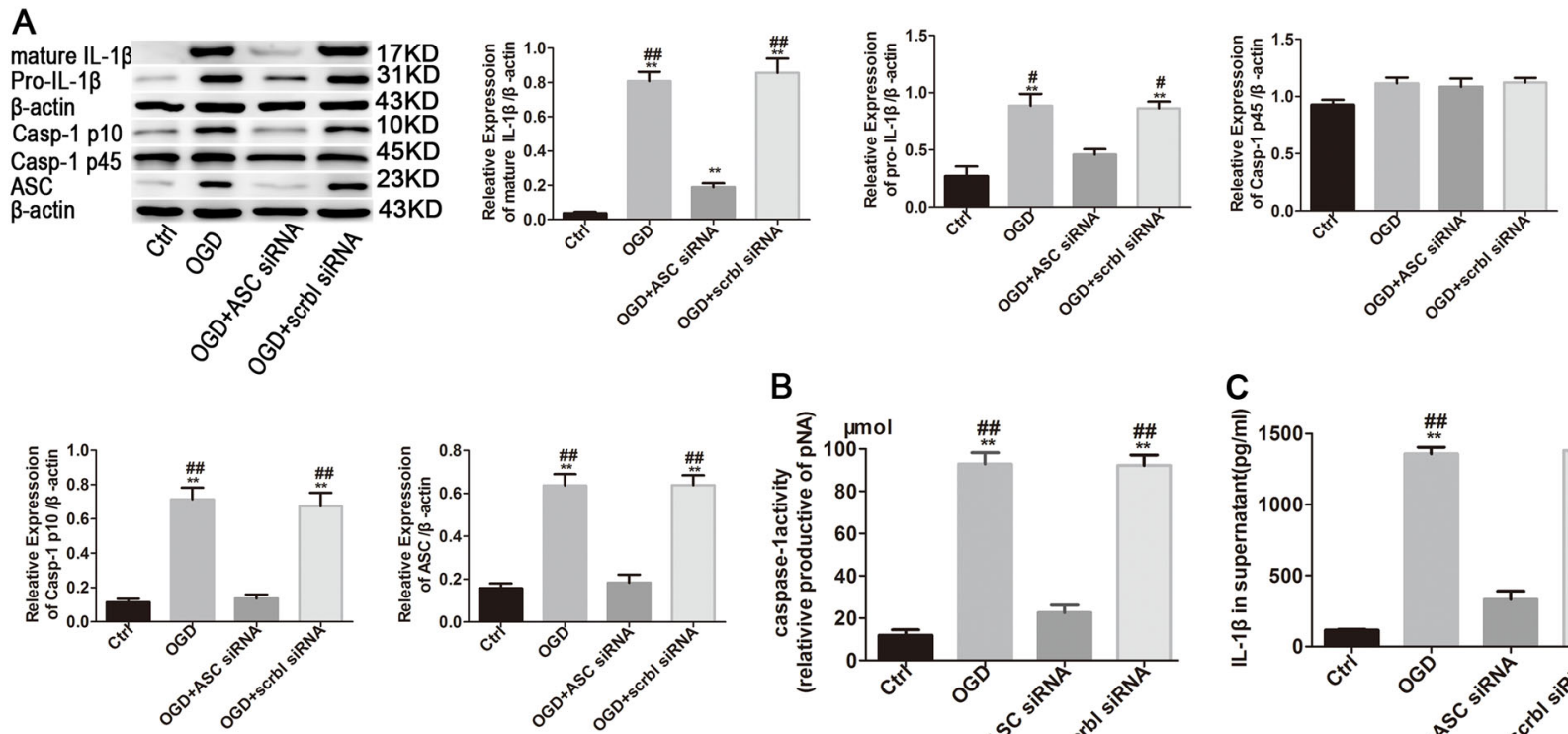

B
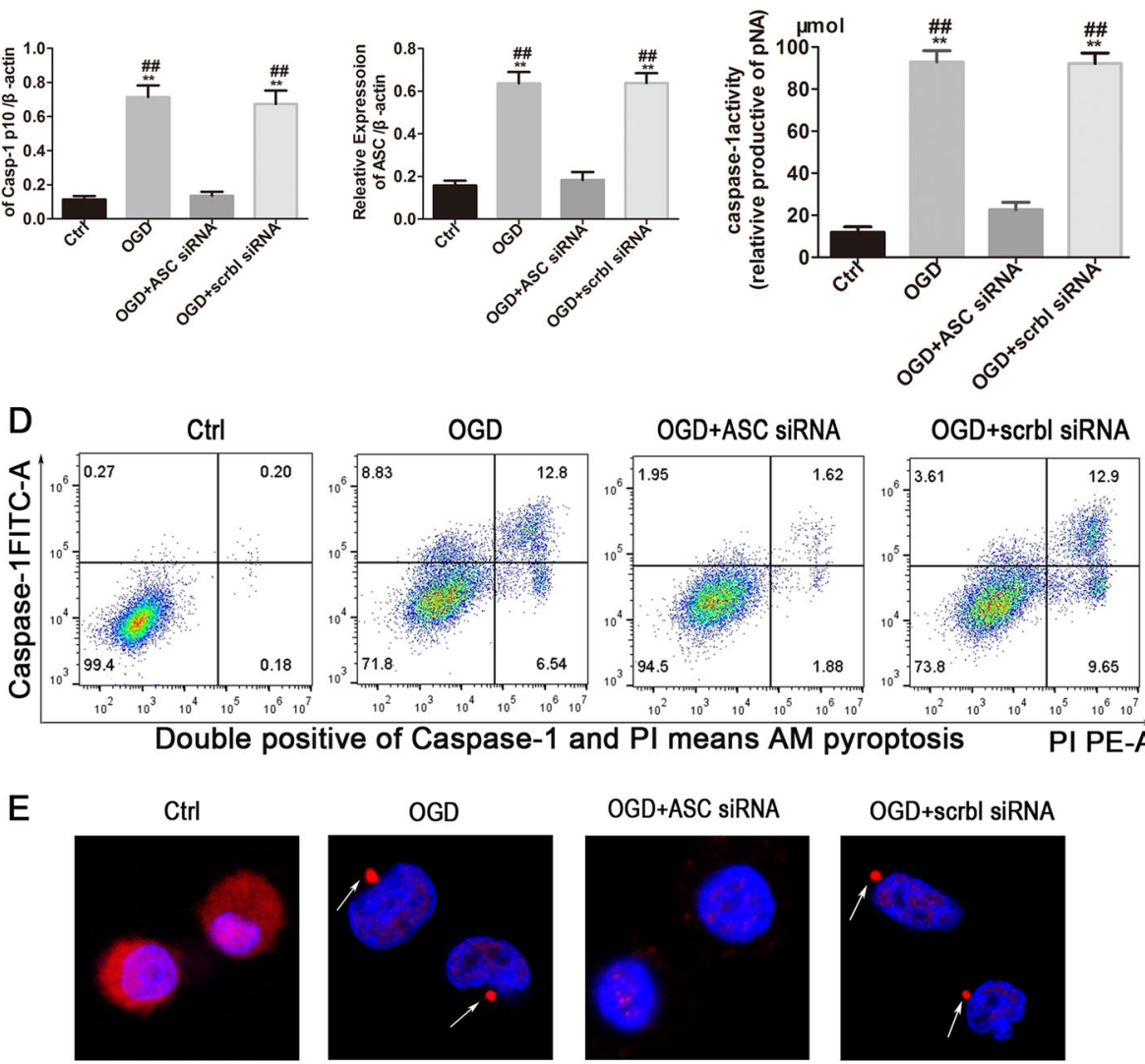

PI PE-A
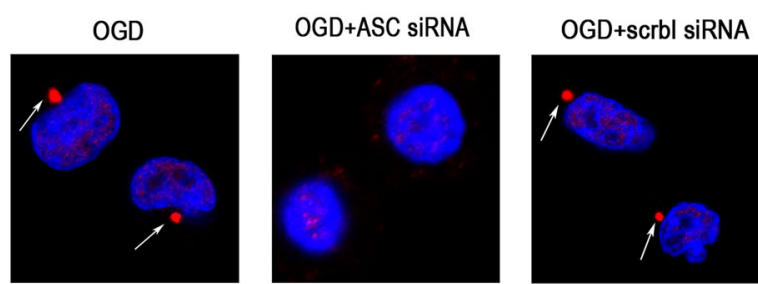

ASC DAPI

*Arrows indicates the cells with ASC foci formation
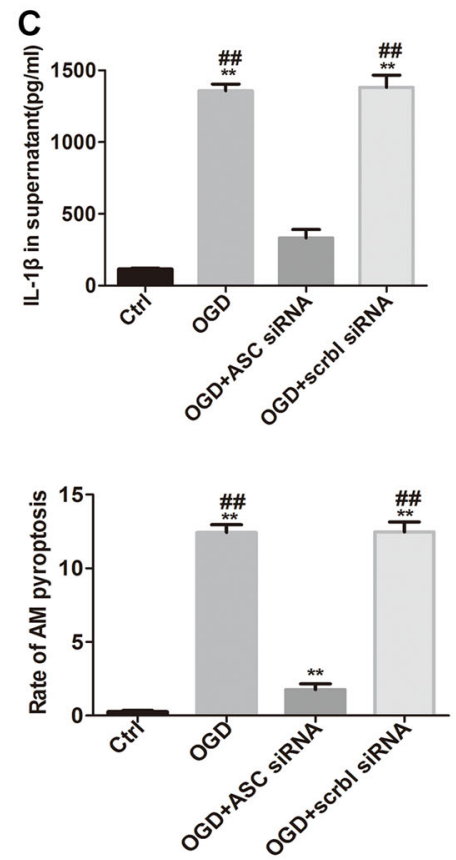

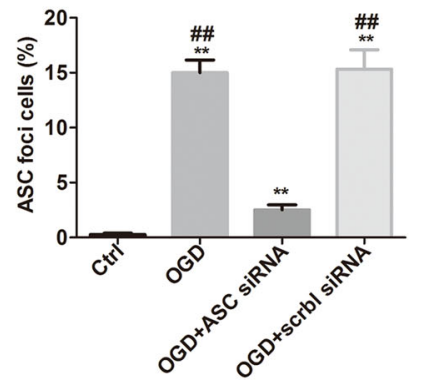

Fig. 2 Knockdown of ASC by specific siRNA mitigates the OGDinduced AM pyroptosis. NR8383 AM were transfected with ASCspecific or scramble (scrbl) siRNA for $48 \mathrm{~h}$, and the cells were exposed to OGD. The relative levels of pyroptosis-related protein expression, caspase- 1 activity, the levels of IL- $1 \beta$ in the supernatants of cultured cells, and the frequency of pyroptotic AM were determined. In addition, the cells were stained with Cy3-anti-ASC (red), and counterstained with DAPI. The frequency of pyroptotic AM was observed by

kits (SHINO-TEST CORP, Kanagawa, Japan and R\&D Systems, Minneapolis, USA), according to the manufacturer's instructions. The experimental and control samples were tested in triplicate simultaneously. The detection limitation is $12 \mathrm{pg} / \mathrm{ml}$ for HMGB 1 and $9 \mathrm{pg} / \mathrm{ml}$ for IL-1 $\beta$. a fluorescent microscope. Data are representative images or expressed as the mean \pm SEM of each group ( $n=3$ per group) from three separate experiments. ${ }^{*} * p<0.01$ vs. the control group. ${ }^{\# \#} p<0.01$ vs. the ASC silenced cells. a The relative levels of pyroptosis-related protein expression. b The caspase- 1 activity. c The levels of IL- $1 \beta$ in the cell supernatants. d The frequency of pyroptotic AM. e The ASC foci formation was analyzed by immunofluorescence (magnification $\times 760)$

\section{Caspase-1 activity}

The caspase- 1 activity in individual samples was determined for the production of $p$ NA using a colorimetric assay kit (Beyotime BIotechology). Briefly, individual cellular and lung tissue protein samples $(50 \mu \mathrm{g} / \mathrm{each})$ were incubated 

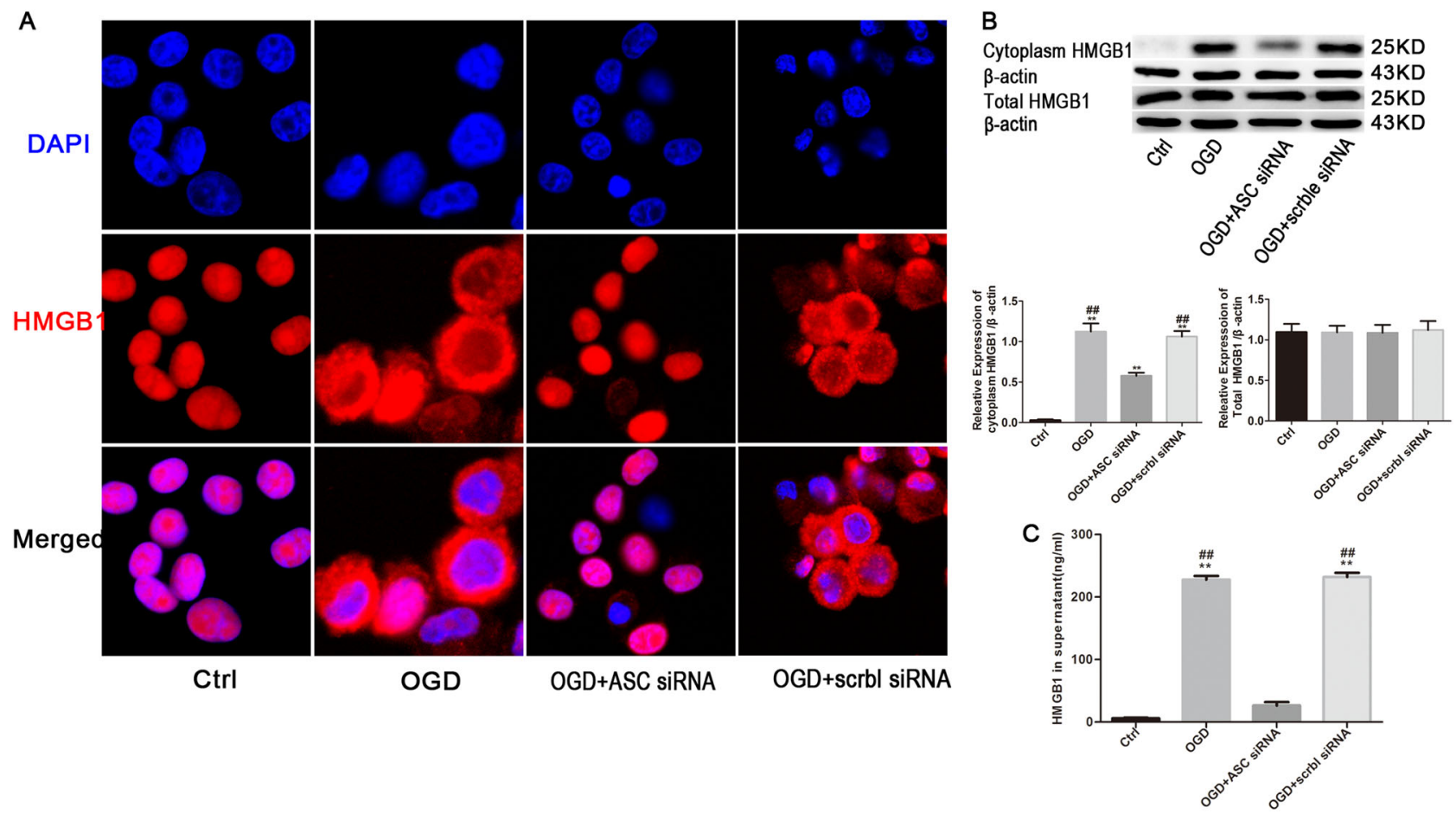

Fig. 3 Inhibiting AM pyroptosis by ASC silencing reduces the OGDenhanced HMGB1 secretion in AM. Following transfection with ASCspecific siRNA or scramble (scrbl) siRNA, the cells were stained with Cy3-anti-HMGB1 and DAPI. The distribution of HMGB1 in the different groups of AM was characterized by confocal microscopy. In addition, the cytosolic and total proteins were extracted, and the relative levels of cytoplasmic and total HMGB1 in AM were determined by Western blot. The levels of HMGB 1 in the cell supernatants

in triplicate with $10 \mathrm{nmol}$ Ac-YVAD-pNA in 96-well microplate at $37^{\circ} \mathrm{C}$ overnight. The caspase- 1 activity in the individual wells was measured for absorbance at $405 \mathrm{~nm}$ using a microplate reader.

\section{Histopathological analysis}

The paraffin-embedded lung tissue sections $(5 \mu \mathrm{m})$ were stained with hematoxylin and eosin (HE). The degrees of lung injury in individual rats were assessed by two pathologists in a blinded manner using a $0-3$ score system ( 0 , normal; 1 , mild; 2 , moderate; 3 , strong) for interstitial/ alveolar edema, hemorrhage, alveolar septal thickening, and infiltration of the inflammatory cells [27].

\section{Statistical analysis}

Data are expressed as means \pm SEM. The difference between the two groups was analyzed by Student $T$ test and the difference among multiple groups was analyzed by ANOVA and post hoc Tukey's multiple comparison test using the Graphpad software. A $p$-value of $<0.05$ was considered statistically significant. were determined by ELISA. Data are representative images or expressed as the mean \pm SEM of each group ( $n=3$ per group) from three separate experiments. a Immunofluorescent characterization of HMGB1 distribution in AM (magnification $\times 740$ ). b Western blot analysis of the levels of cytoplasmic and total HMGB1 expression in AM. $\mathbf{c}$ The levels of HMGB 1 in the supernatants. ${ }^{* *} p<0.01$ vs. the control group; ${ }^{\# \#} p<0.01$ or ${ }^{\#} p<0.05$ vs. the OGD + ASC siRNA group

\section{Results}

\section{Both OGD and CPB induce AM pyroptosis}

Caspase- 1 can be activated and cleaved into two fragments (caspase-1 p10 and p20), via ASC adaptor, inducing cell pyroptosis $[28,29]$. Pro-IL-1 $\beta$ (p35) can be cleaved by activated capase- 1 to produce mature IL-1 $\beta$ (p17) during cell pyroptosis [12]. To evaluate whether OGD induced AM pyroptosis, AM cells were exposed to OGD or cultured in complete medium (control) for $6 \mathrm{~h}$ and the relative levels of caspase-1, cleaved caspase-1 p10, pro-IL-1 $\beta$, mature IL-1 $\beta$, and ASC expression in individual groups of cells were determined by Western blot. As shown in Fig. 1a, OGD exposure significantly increased the relative levels of cleaved caspase- 1 p10, pro-IL-1 $\beta$, mature IL- $1 \beta$, and ASC ( $p<0.01$ for all), but not caspase-1 p45 expression in AM. Furthermore, OGD exposure significantly increased the levels of caspase-1 activity in AM and the percentages of pyroptotic AM in vitro $(p<0.01$ for all, Fig. 1 b, c). Similarly, in comparison with that in the lungs of the sham group, the $\mathrm{CPB}$ procedure also significantly increased the relative levels of pro-IL-1 $\beta$, mature IL- $1 \beta$, caspase-1 p10, 
A
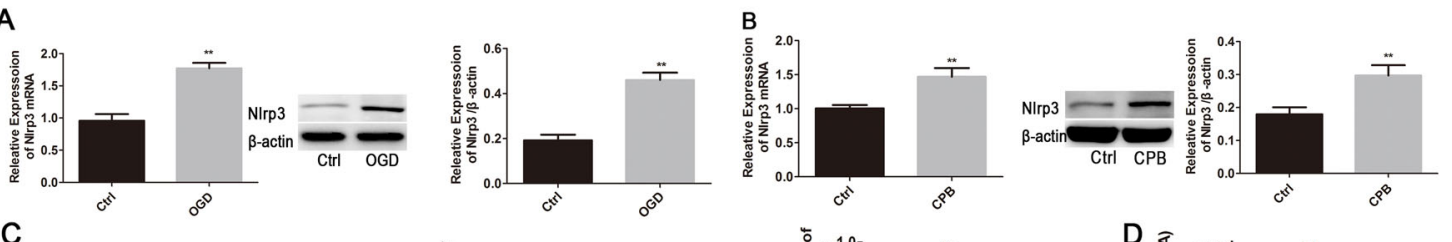

C
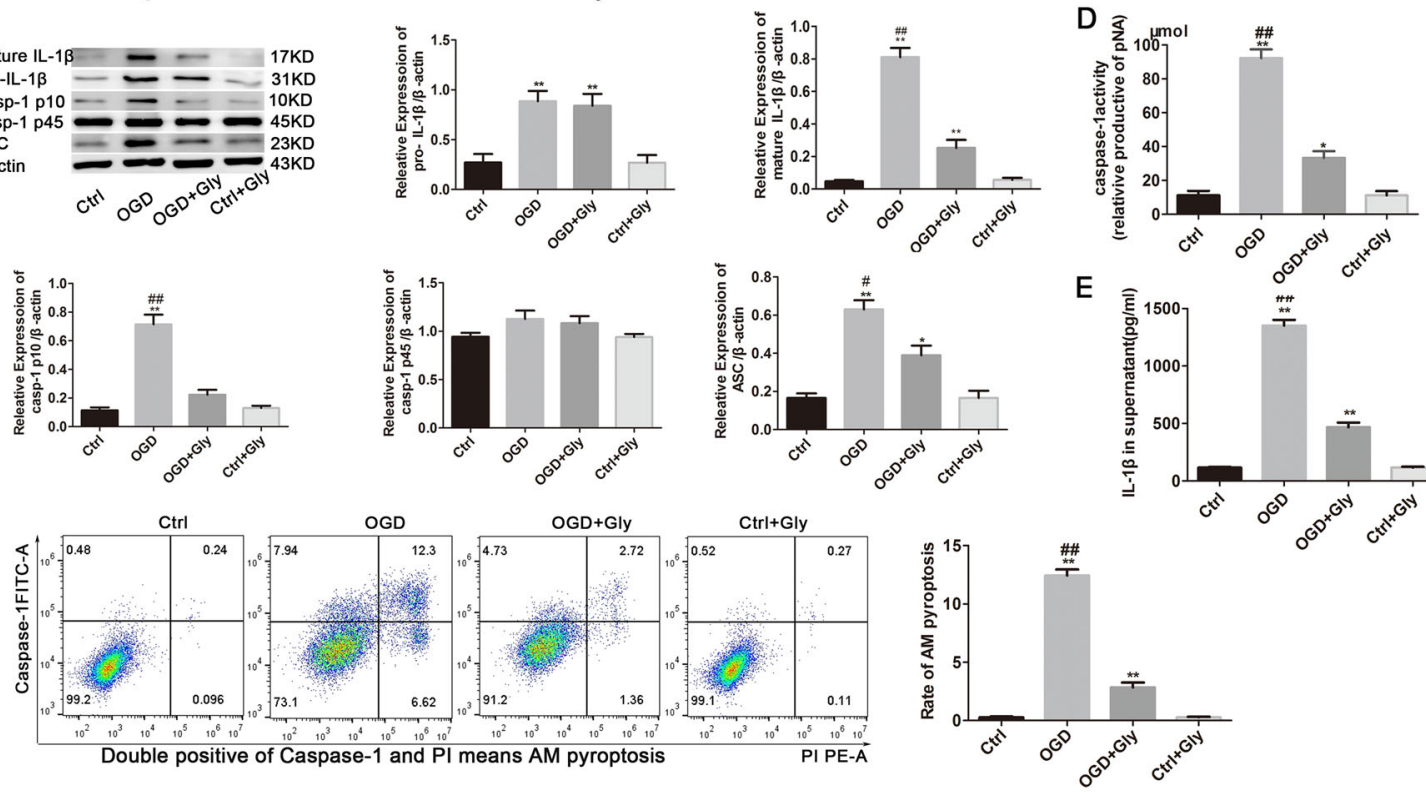

G
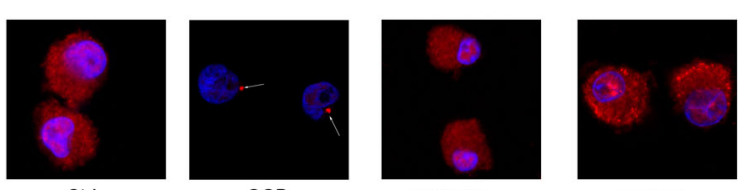

ASC DAPI *Arrows indicates the cells with ASC foci formation
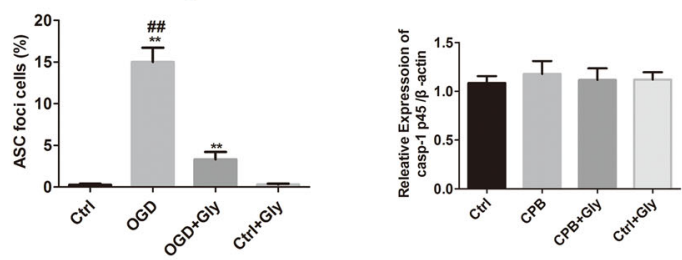
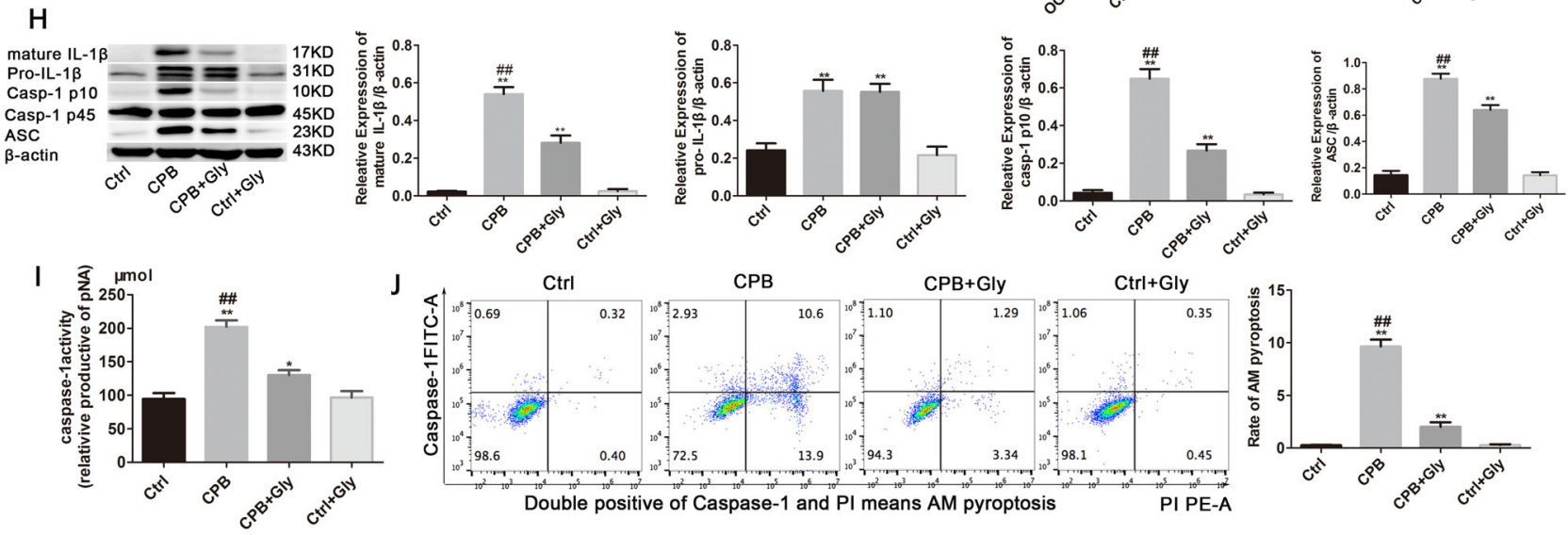

Fig. 4 Inhibition of Nlrp3-related inflammation by glyburide attenuates the OGD-induced and CPB-induced pyroptosis in AM. NR8383 AM were pre-treated with, or without, glyburide for $30 \mathrm{~min}$ and exposed to OGD. In addition, rats were randomized and treated intraperitoneally with a single dose of $50 \mathrm{mg} / \mathrm{kg}$ glyburide $1 \mathrm{~h}$ before the CPB or sham surgery. After $6 \mathrm{~h}$, the levels of Nlrp3 expression and AM pyroptosis were determined. Data are representative images or expressed as the mean \pm SEM of each group $(n=3$ per group for cell assays; $n=6$ per group of rats) from three separate experiments. $\mathbf{a}, \mathbf{b}$
Quantitative RT-PCR and Western blot analysis of the levels of Nlrp3 expression in AM and in the lungs. $\mathbf{c}, \mathbf{h}$ Western blot analysis of the relative levels of pyroptosis-related protein expression in AM and in the lungs, respectively. $\mathbf{d}$, i The levels of caspase- 1 activity in AM and the lungs, respectively. $\mathbf{e}$ The levels of IL- $1 \beta$ in the cell supernatants. f, $\mathbf{j}$ The frequency of pyroptotic AM and BALF AM. $\mathbf{g}$ The ASC foci formation in $\mathrm{AM}$ was analyzed by immunofluorescence (magnification $\times 760)$. ${ }^{* *} p<0.01$ or $* p<0.05$ vs. the control group; ${ }^{\# \#} p<0.01$ vs. the $\mathrm{OGD}+$ glyburide (gly) group or the $\mathrm{CPB}+$ glyburide (gly) group 

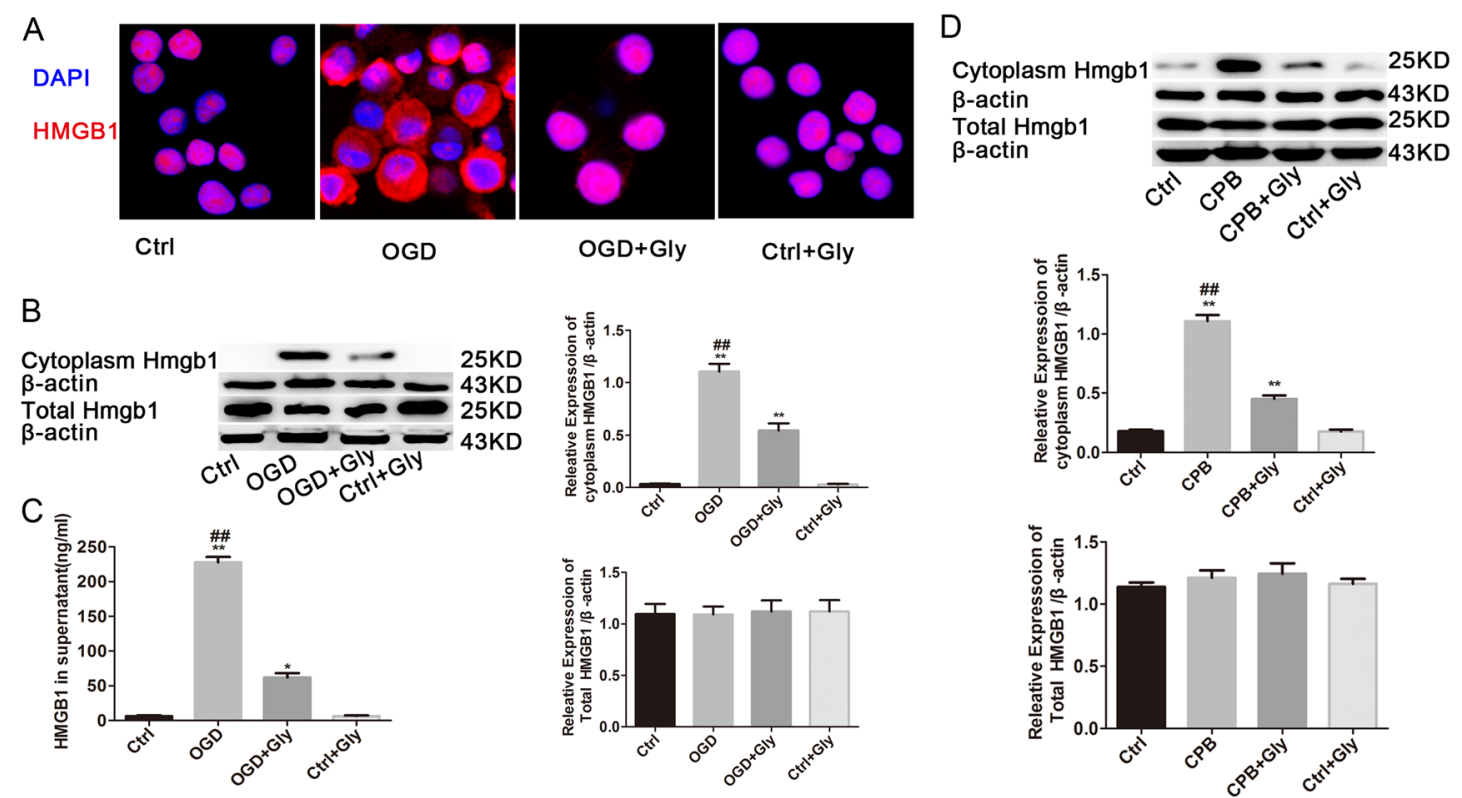

Fig. 5 Inhibition of Nlrp3-mediated pyroptosis by glyburide reduces HMGB1 release from AM. NR8383 AM were pre-treated with, or without, glyburide for $30 \mathrm{~min}$ and exposed to OGD. In addition, rats were randomized and treated intraperitoneally with a single dose of 50 $\mathrm{mg} / \mathrm{kg} 1 \mathrm{~h}$ before CPB or sham surgery. After $6 \mathrm{~h}$, the different groups of AM NR8383 were stained with Cy3-550-anti-HMGB1 and DAPI, and characterized by confocal microscopy. The levels of cytoplasmic and total HMGB1 expression, and HMGB1 in the cell supernatants were determined. a Immunoflurescent analysis of HMGB1 distribution

and ASC, but not caspase- $1 \mathrm{p} 45$ expression in the lungs of rats $(p<0.01$ for all, Fig. 1d). The CPB procedure significantly increased the caspase-1 activity in the lungs of rats and the percentages of pyroptotic AM in the BALF of rats $(p<0.01$ for all, Fig. 1d, f). Together, such data indicated that OGD exposure and CPB procedure induced AM pyroptosis in vitro and in vivo.

\section{Knockdown of ASC by specific siRNA mitigates the OGD-induced AM pyroptosis}

ASC is crucial for inflammasome-induced caspase- 1 activation and pyroptosis. To investigate the potential role of ASC in OGD-induced AM pyroptosis, AM cells were transfected with, or without, control or ASC-specific siRNA for $48 \mathrm{~h}$ and exposed to OGD. The levels of AM pyroptosis were determined. Transfection with ASC-specific siRNA, but not control siRNA, significantly reduced the levels of ASC expression in AM. Knockdown of ASC significantly mitigated the OGD-upregulated pro-IL-1 $\beta$, mature IL-1 $\beta$, and cleaved caspase- 1 p10 expression, but did not affect the caspase-1 p45 expression in AM (Fig. 2a). Similarly, ASC silencing decreased the OGD-enhanced caspase-1 activity and IL-1 $\beta$ secretion in AM $(p<0.01$ for all, Fig. $2 b, c)$. ASC silencing significantly reduced the OGD-stimulated in the cultured AM (magnification $\times 740$ ). $\mathbf{b}$, $\mathbf{d}$ Western blot analysis of the levels of cytoplasmic and total HMGB1 in the cultured AM and BALF AM, respectively. c HMGB1 levels in the cell supernatants. Data are representative images or expressed as the mean \pm SEM of each group ( $n=3$ per group for cell assays; $n=6$ per group of rats) from three separate experiments. ${ }^{* *} p<0.01$ vs. the control group; ${ }^{\# \#} p$ $<0.01$ or ${ }^{\#} p<0.05$ vs. the OGD + glyburide (gly) group or the CPB + glyburide (gly) group

pyroptosis in AM ( $p<0.01$ for all, Fig. 2d, e). The ASC foci formation is crucial for caspase-1 activation and consequent cell pyroptosis [30]. While the ASC foci formation was observed in the OGD-exposed cells, it was absent in the OGD-exposed ASC-silencing AM (Fig. 2e). Hence, knockdown of ASC mitigated the OGD-triggered pyroptosis in AM, indicating that ASC/caspase-1 activation mediated AM pyroptosis.

\section{Inhibiting AM pyroptosis by ASC silencing reduces the OGD-enhanced HMGB1 secretion in AM}

To analyze how AM pyroptosis affected HMGB1 secretion, we studied whether knockdown of ASC affected the OGD-enhanced HMGB1 expression, cytoplasmic translocation, and extracellular release in AM, given that the translocation of HMGB1 from the nucleus to cytoplasm is the first step for its extracellular secretion [31]. We found that HMGB1 was mainly located in the nucleus of the control macrophage, consistent with other studies [32, 33]. OGD exposure induced HMGB1 translocation to the cytoplasm, which was significantly abrogated in the ASC-silencing AM (Fig. 3a). In addition, we measured the cytoplasmic and total HMGB1 protein levels in the different groups of AM, and we found that in comparison 
A
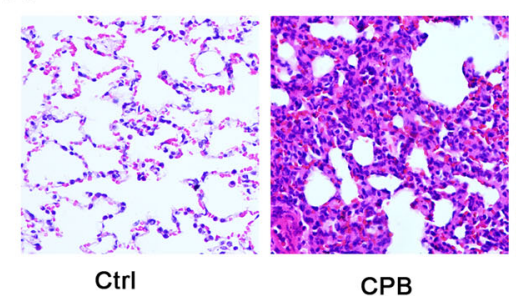

CPB

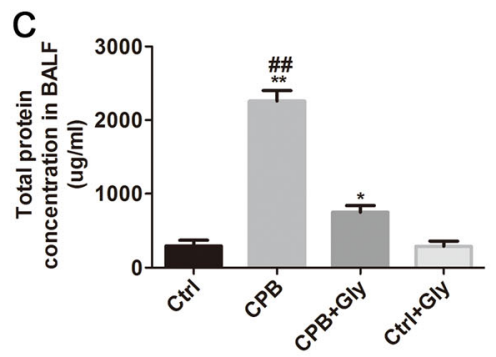

$\mathrm{F}$

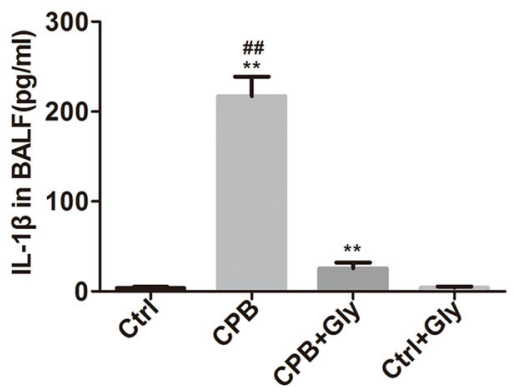

Fig. 6 Inhibition of Nlrp3-related inflammation by glyburide reduces the CPB-induced ALI in rats. Rats were randomized and treated intraperitoneally with a single dose of $50 \mathrm{mg} / \mathrm{kg} 1 \mathrm{~h}$ before CPB or sham surgery. After $6 \mathrm{~h}$, the lung tissue sections were stained with HE, and the severity of lung injury was scored. The levels of serum and BALF IL-1 $\beta$ and HMGB1 were determined by ELISA. Data are
B
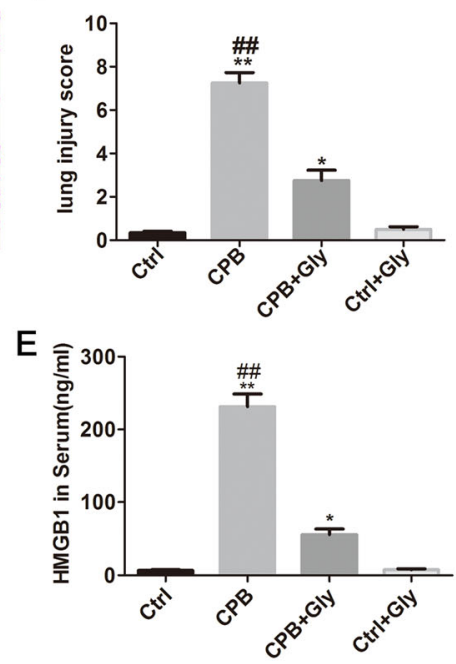

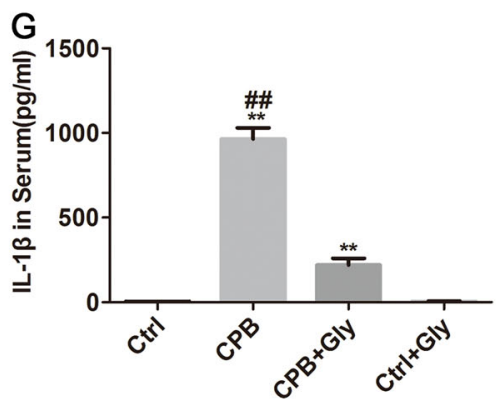

representative images (magnification $\times 200$ ) or expressed as the mean \pm SEM of each group ( $n=6$ per group) from three separate experiments. a Histological examination. b The lung injury scores. c The levels of total proteins in BALF. $\mathbf{d}-\mathbf{g}$ The levels of serum and BALF IL-1 $\beta$ and HMGB1. $* * p<0.01$ vs. the control group; ${ }^{\# \#} p<0.01$ or ${ }^{\#} p$ $<0.05$ vs. the $\mathrm{CPB}+$ glyburide (gly) group

of Nlrp3 mRNA transcripts and protein expression in AM and the lung tissues of rats, respectively $(p<0.01$ for all, Fig. $4 a, b)$. Pretreatment with glyburide $(200 \mu \mathrm{m})$ [35] did not significantly change the levels of pro-IL- $1 \beta$, IL- $1 \beta$, caspase-1 p10 and ASC expression, caspase-1 activity, IL-1 $\beta$ secretion, and the frequency of pyroptotic control AM. In contrast, the same treatment significantly mitigated the OGD-upregulated expression of these molecules, caspase-1 activity, IL-1 $\beta$ secretion, and the percentages of pyroptotic AM, but not caspase-1 $\mathrm{p} 45$ expression in AM $(p<0.05$ for all, Fig. 4c-f). In addition, inhibition of Nlrp3-related inflammasomes by glyburide also inhibited the OGD-induced ASC foci formation in AM (Fig. 4g). Further determining the importance of Nlrp3 in the CPB-induced pyroptosis of AM indicated that pre-treatment with glyburide $(50 \mathrm{mg} / \mathrm{kg})$ significantly attenuated the CPBincreased ASC, caspase-1 p10, mature IL-1 $\beta$ expression, caspase-1 activity, and the frequency of BALF pyroptotic $\mathrm{AM}$, but not pro-IL-1 $\beta$, and caspase-1 p45 expression in the lung tissue of rats $(p<0.01$ for all, Fig. $4 \mathrm{~h}-\mathrm{j})$. 
These data indicated that Nlrp3/ASC/caspase-1 activation was crucial for OGD-induced and CPB-induced AM pyroptosis.

\section{Inhibition of NIrp3-mediated pyroptosis by glyburide reduces HMGB1 release from AM}

To determine whether inhibition of Nlrp3-mediated AM pyroptosis could reduce HMGB1 release, we assessed the HMGB1 protein levels, intracellular translocation, and extracellular release in AM. Confocal microscopy showed that after AM was exposed to OGD, most HMGB1 were translocated from the nucleus to the cytoplasm of the AM. Inhibition of Nlrp3 by glyburide attenuated the cytoplasmic translocation of HMGB1 and significantly reduced the levels of cytoplasmic HMGB1 in AM (Fig. 5a). Although the total HMGB1 protein levels were not changed in these four groups of AM, the cytoplasmic HMGB1 levels increased significantly in the OGD group, which were significantly reduced in the cells treated with glyburide (Fig. 5b). In addition, HMGB1 levels in the supernatants of cultured OGD-exposed AM were also reduced by glyburide treatment (Fig. 5c). Furthermore, we examined whether glyburide treatment could also decrease HMGB1 release in different rat groups by western blotting. Although there was no significant difference in the levels of total HMGB1 expression among the four groups. The levels of cytoplasmic HMGB1 protein in the CPB group were significantly decreased by glyburide treatment (Fig. 5d). Such data indicated that inhibition of Nlrp3-mediated AM pyroptosis by glyburide reduced HMGB1 release from the AM.

\section{Inhibition of NIrp3-related inflammation by glyburide reduces the CPB-induced ALI in rats}

Finally, we evaluated the impact of glyburide treatment on the CPB-induced ALI in rats. Pre-treatment with glyburide did not significantly alter the lung morphology, the levels of BALF total proteins, IL-1 $\beta$ and HMGB1, as well as the levels of serum IL-1 $\beta$ and HMGB1 in the control rats (Fig. 6). While the CPB rats displayed alveolar septal thickening, interstitial edema, neutrophil infiltration, and erythrocyte effusion, as well as high lung injury scores, pretreatment with glyburide significantly reduced the lung morphological changes and lung injury scores in the CPB rats. In comparison with that in the $\mathrm{CPB}$ rats, pre-treatment with glyburide significantly decreased the levels of BALF total proteins, IL-1 $\beta$ and HMGB1, as well as serum IL-1 $\beta$ and HMGB1 the CPB rats $(p<0.01$ for all, Fig. $6 c-g)$. Collectively, these results demonstrated that inhibition of Nlrp3 inflammasomes by glyburide reduced the CPBinduced ALI in rats.

\section{Discussion}

ALI and its more severe form, acute respiratory distress syndrome (ARDS) have a mortality of more than $40 \%$ [36, 37]. The abnormal secretion of HMGB1 during CPB enhances the ALI [38]. However, the mechanisms by which CPB promotes HMGB1 secretion during the process of ALI has not been clarified. Recent studies have showed that AM pyroptosis exaggerates lung inflammation and inhibiting LPS-mediated AM pyroptosis reduces lung injury in a model of LPS-induced ALI [39]. In addition, unlike apoptosis, which depends on the release of no inflammatory factors, pyroptosis depends on caspase- 1 activation, leading to concomitant release of inflammatory factors, such as IL$1 \beta$ and IL-18, which are known to be closely associated with ALI [40, 41]. Different from the LPS-induced ALI, the CPB-induced ALI is attributed to lung I/R [2]. Despite tremendous advances in organ preservation during the perioperative period, ALI remains a serious clinical complication of $\mathrm{CPB}$, causing a mortality rate of $15-50 \%$ [42, 43]. In this study, we isolated AM from BLAF of CPB rats and found that OGD or CPB induced AM pyroptosis. However, it is unclear about the role of AM pyroptosis in CPB-induced ALI. Firstly, we investigated the mechanism by which OGD induced AM pyroptosis in vitro. As an adapter protein, ASC is indispensable for inducing pyroptosis [44]. The pyroptosome of ASC oligomer is required for caspase-1 activation and pyroptosis induction [9]. Indeed, we found that transfection with the ASC-specific siRNA not only dramatically reduced the levels of ASC expression, but also mitigated the OGD-induced capase-1 activation, IL-1 $\beta$ secretion and the percentages of AM pyroptosis in AM. Our findings support the notion that ASC is crucial for the Nlrp3 inflammasome-mediated caspase-1 activation and AM pyroptosis [28, 45, 46].

Nlrp3 can, through the toll-like receptor (TLR), recognize damage-associated molecular patterns (DAMPs) released from necrotic cells during the process of I/R injury [47, 48]. Nlrp3 can be activated by engagement of TLR and other sensing danger signals to activate the NF-kB signaling, leading to upregulated Nlrp3 and IL-1 $\beta$ expression in AM [41, 49, 50]. In this study, we found that OGD exposure and CPB significantly upregulated Nlrp3 expression in AM, suggesting that upregulated Nlrp3 expression may mediate AM pyroptosis following the CPB. Furthermore, we found that pre-treatment with glyburide to inhibit the Nlrp3 activation significantly mitigated the OGD and CPBinduced AM pyroptosis, consistent with previous findings [24, 51]. Similarly, ASC silencing also significantly reduced the OGD exposure-induced AM pyroptosis, indicating that ASC was indispensable in mediating AM pyroptosis. We recognized that while ASC silencing, but not pre-treatment with glyburide to inhibit the Nlrp3, significantly reduced the 
levels of pro-IL-1 $\beta$ in AM although both effectively decreased IL-1 secretion in AM. The different effects between ASC silencing and glyburide pre-treatment may stem from that ASC silencing decreased the NF-kB signaling, leading to lower levels of pro-IL-1 $\beta$ expression [21, 52]. In contrast, Nlrp3 inhibition by Glyburide mainly affected the caspase- 1 mediated cleavage of pro-IL- $1 \beta$, but did not affect pro-IL-1 $\beta$ expression in AM in a hypoxic and malnutrition condition [24]. However, the precise mechanisms underlying the difference remain to be determined. CPB can induce inflammation and inflammatory IL-1 $\beta$ production [53]. We found that inhibition of AM pyroptosis also significantly reduced the OGD exposure or CPBinduced IL-1 $\beta$ production in AM, which may alleviate inflammatory responses following the CPB.

Collectively, our findings indicate that Nlrp3/ASC triggers AM pyroptosis. We are interested in further investigating the importance of ASC in the Nlrp3-medaited AM pyroptosis in vivo if suitable ASC inhibitor and ASC knockout rats are available.

HMGB1, one of the DAMPs, is an important inflammatory factor and involved in the pathogenesis of inflammatory disorders, such as I/R-induced injury [54-57]. Extracellular HMGB1 can engage the TLR2, TLR4 and RAGE to trigger $\mathrm{I} / \mathrm{R}$ inflammatory responses $[58,59]$. In this study, we found that inhibition of AM pyroptosis by ASC silencing or glyburide treatment significantly reduced the OGD-promoted HMGB1 release from AM. Similarly, treatment with glyburide also significantly inhibited the CPB-induced cytoplasmic transportation of HMGB1 in AM. Such data demonstrated that Nlrp3/ASC-mediated AM pyroptosis induced HMGB1 secretion from AM. However, OGD exposure or CPB increased pyroptosis and HMGB1 secretion may be independent of ASC given that ASC is dispensable for NLRC4 or NLRP1b inflammasomemediated pyroptosis [60]. Recent studies have shown that Salmonella typhimurium-infected macrophages secrete high levels of HMGB1 in vitro, which was dependent on caspase-1 and NLRC4, but independent of ASC [61]. Similarly, ASC-deficient mice produced high levels of serum HMGB1 after challenged with LeTx [62]. Hence, it is possible that CPB may lead to inflammasome activation and high levels of HMGB1 secretion, independent of ASC. We are interested further investigating whether OGD exposure or $\mathrm{CPB}$ can also induce pyroptosis and HMGB1 secretion in ASC-/- AM or rats. Given that the secreted HMGB1 can enhance the Nlrp3 activation and further promote caspase-1 activation and pyroptosis [63, 64], the increased levels of HMGB1 may feedbackpositively enhance AM pyroptosis, exacerbating the inflammatory response and ALI. Actually, we have found that inhibition of Nlrp3/ASC-mediated pyroptosis significantly reduced the CPB-related ALI in rats.
Interestingly, Nlrp3 inflammation contributes to the pathogenesis of ventilator-induced lung injury and is essential for paraquat-induced ALI $[35,65]$. The significant reduction of HMGB1 secretion and ALI by inhibition of AM pyroptosis suggests that inhibition of AM pyroptosis may be a new therapeutic strategy for prevention and intervention of ALI. Therefore, our findings may provide new insights into the pathogenesis of CPB-related ALI and aid in design of new therapies for CPB-related and other types of ALI.

In conclusion, our study in vivo and in vitro demonstrated that Nlrp3/ASC-mediated AM pyroptosis enhanced HMGB1 release, which induced I/R-related ALI following the CPB. Inhibition of AM pyroptosis may be a potential therapy for the I/R-related ALI.

Funding This work was supported by the National Natural Science Foundation of China (NSFC, NO.81370178 and 81570068)

\section{Compliance with ethical standards}

Conflict of interest The authors declare that they have no conflict of interest.

\section{References}

1. Zhao M, Wu B, Yang S, et al. Analysis of cardiopulmonary bypass status in China: eight-year development trends. Perfusion. 2013;28:21-25.

2. Apostolakis E, Filos KS, Koletsis E, et al. Lung dysfunction following cardiopulmonary bypass. J Card Surg. 2010;25:47-55.

3. Nicolls MR, Laubach VE. Traumatic brain injury: lungs in a RAGE. Sci Transl Med. 2014;6:252fs234.

4. Andersson U, Tracey KJ. HMGB1 is a therapeutic target for sterile inflammation and infection. Annu Rev Immunol. 2011;29:139-62.

5. Yang Z, Deng Y, Su D, et al. TLR4 as receptor for HMGB1mediated acute lung injury after liver ischemia/reperfusion injury. Lab Invest. 2013;93:792-800.

6. Ge J, Tian J, Yang H, et al. Alpha7 Nicotine acetylcholine receptor agonist PNU-282987 attenuates acute lung injury in a cardiopulmonary bypass model in rats. Shock. 2017;47: 474-9.

7. Deng Y, Yang Z, Gao Y, et al. Toll-like receptor 4 mediates acute lung injury induced by high mobility group box-1. PLoS One. 2013;8:e64375.

8. Miao EA, Rajan JV, Aderem A. Caspase-1-induced pyroptotic cell death. Immunol Rev. 2011;243:206-14.

9. Labbe K, Saleh M. Cell death in the host response to infection. Cell Death Differ. 2008;15:1339-49.

10. Bergsbaken T, Fink SL, Cookson BT. Pyroptosis: host cell death and inflammation. Nat Rev Microbiol. 2009;7:99-109.

11. Schroder K, Muruve DA, Tschopp J. Innate immunity: cytoplasmic DNA sensing by the AIM2 inflammasome. Curr Biol. 2009; 19:R262-265.

12. Duprez L, Wirawan E, Vanden Berghe T, et al. Major cell death pathways at a glance. Microbes Infect. 2009;11:1050-62.

13. Antonopoulos C, Russo HM, El Sanadi C, et al. Caspase-8 as an effector and regulator of NLRP3 inflammasome signaling. J Biol Chem. 2015;290:20167-84.

14. Kayagaki N, Warming S, Lamkanfi M, et al. Non-canonical inflammasome activation targets caspase-11. Nature. 2011;479:117-21. 
15. Kayagaki N, Stowe IB, Lee BL, et al. Caspase-11 cleaves gasdermin $\mathrm{D}$ for non-canonical inflammasome signalling. Nature. 2015;526:666-71.

16. Arnold IC, Zhang X, Urban S, et al. NLRP3 controls the development of gastrointestinal CD11b(+) dendritic cells in the steady state and during chronic bacterial infection. Cell Rep. 2017;21:3860-72.

17. Bruchard M, Rebe C, Derangere V, et al. The receptor NLRP3 is a transcriptional regulator of $\mathrm{TH} 2$. Differentiation. 2015;16:859-70.

18. Guo Z, Yu S, Chen X, et al. NLRP3 is involved in ischemia/ reperfusion injury. CNS Neurol Disord Drug Targets. 2016;15:699-712.

19. Mezzaroma E, Toldo S, Farkas D, et al. The inflammasome promotes adverse cardiac remodeling following acute myocardial infarction in the mouse. Proc Natl Acad Sci USA. 2011;108:19725-30.

20. Shigeoka AA, Mueller JL, Kambo A, et al. An inflammasomeindependent role for epithelial-expressed Nlrp3 in renal ischemiareperfusion injury. J Immunol. 2010;185:6277-85.

21. Kamo N, Ke B, Ghaffari AA, et al. ASC/caspase-1/IL-1beta signaling triggers inflammatory responses by promoting HMGB1 induction in liver ischemia/reperfusion injury. Hepatology. 2013;58:351-62.

22. Xu J, Jiang Y, Wang J, et al. Macrophage endocytosis of highmobility group box 1 triggers pyroptosis. Cell Death Differ. 2014;21:1229-39.

23. Su DS, Wang XR, Zheng YJ, et al. Retrograde cerebral perfusion of oxygenated, compacted red blood cells attenuates brain damage after hypothermia circulation arrest of rat. Acta Anaesthesiol Scand. 2005;49:1172-81.

24. Lamkanfi M, Mueller JL, Vitari AC, et al. Glyburide inhibits the cryopyrin/Nalp3 inflammasome. J Cell Biol. 2009;187:61-70.

25. Choi G, Wolthuis EK, Bresser $P$, et al. Mechanical ventilation with lower tidal volumes and positive end-expiratory pressure prevents alveolar coagulation in patients without lung injury. Anesthesiology. 2006;105:689-95.

26. Zhang W, Xu X, Kao R, et al. Cardiac fibroblasts contribute to myocardial dysfunction in mice with sepsis: the role of NLRP3 inflammasome activation. PLoS One. 2014;9:e107639.

27. McGuigan RM, Mullenix P, Norlund LL, et al. Acute lung injury using oleic acid in the laboratory rat: establishment of a working model and evidence against free radicals in the acute phase. Curr Surg. 2003;60:412-7.

28. Fang R, Tsuchiya K, Kawamura I, et al. Critical roles of ASC inflammasomes in caspase-1 activation and host innate resistance to Streptococcus pneumoniae infection. J Immunol. 2011;187: 4890-9.

29. Srinivasula SM, Poyet JL, Razmara M, et al. The PYRIN-CARD protein ASC is an activating adaptor for caspase-1. J Biol Chem. 2002;277:21119-22.

30. Stehlik C, Lee SH, Dorfleutner A, et al. Apoptosis-associated specklike protein containing a caspase recruitment domain is a regulator of procaspase-1 activation. J Immunol. 2003;171:6154-63.

31. Scaffidi P, Misteli T, Bianchi ME. Release of chromatin protein HMGB1 by necrotic cells triggers inflammation. Nature. 2002;418:191-5.

32. Wang H, Liao H, Ochani M, et al. Cholinergic agonists inhibit HMGB1 release and improve survival in experimental sepsis. Nat Med. 2004;10:1216-21.

33. Rendon-Mitchell B, Ochani M, Li J, et al. IFN-gamma induces high mobility group box 1 protein release partly through a TNFdependent mechanism. J Immunol. 2003;170:3890-7.

34. Vajjhala PR, Mirams RE, Hill JM. Multiple binding sites on the pyrin domain of ASC protein allow self-association and interaction with NLRP3 protein. J Biol Chem. 2012;287:41732-43.
35. Liu Z, Zhao H, Liu W, et al. NLRP3 inflammasome activation is essential for paraquat-induced acute lung injury. Inflammation. 2015;38:433-44.

36. Matthay MA, Zimmerman GA, Esmon C, et al. Future research directions in acute lung injury: summary of a National Heart, Lung, and Blood Institute working group. Am J Respir Crit Care Med. 2003;167:1027-35.

37. Wheeler AP, Bernard GR. Acute lung injury and the acute respiratory distress syndrome: a clinical review. Lancet. 2007;369:1553-64.

38. Liu X, Cao H, Li J, et al. Autophagy induced by DAMPs facilitates the inflammation response in lungs undergoing ischemiareperfusion injury through promoting TRAF6 ubiquitination. Cell Death Differ. 2017;24:683-93.

39. Wu DD, Pan PH, Liu B, et al. Inhibition of alveolar macrophage pyroptosis reduces lipopolysaccharide-induced acute lung injury in mice. Chin Med J. 2015;128:2638-45.

40. Kovarova M, Hesker PR, Jania L, et al. NLRP1-dependent pyroptosis leads to acute lung injury and morbidity in mice. J Immunol. 2012;189:2006-16.

41. Mao K, Chen S, Chen M, et al. Nitric oxide suppresses NLRP3 inflammasome activation and protects against LPS-induced septic shock. Cell Res. 2013;23:201-12.

42. Rubenfeld GD, Caldwell E, Peabody E, et al. Incidence and outcomes of acute lung injury. N Engl J Med. 2005;353:1685-93.

43. Clark SC. Lung injury after cardiopulmonary bypass. Perfusion. 2006;21:225-8.

44. Franchi L, Stoolman J, Kanneganti TD, et al. Critical role for Ipaf in Pseudomonas aeruginosa-induced caspase-1 activation. Eur J Immunol. 2007;37:3030-9.

45. Hornung V, Ablasser A, Charrel-Dennis M, et al. AIM2 recognizes cytosolic dsDNA and forms a caspase-1-activating inflammasome with ASC. Nature. 2009;458:514-8.

46. Compan V, Martin-Sanchez F, Baroja-Mazo A, et al. Apoptosisassociated speck-like protein containing a CARD forms specks but does not activate caspase-1 in the absence of NLRP3 during macrophage swelling. J Immunol. 2015;194:1261-73.

47. Eltzschig HK, Eckle T. Ischemia and reperfusion-from mechanism to translation. Nat Med. 2011;17:1391-401.

48. Fann DY, Lee SY, Manzanero S, et al. Pathogenesis of acute stroke and the role of inflammasomes. Ageing Res Rev. 2013;12:941-66.

49. Martinon F, Mayor A, Tschopp J. The inflammasomes: guardians of the body. Annu Rev Immunol. 2009;27:229-65.

50. Qi Y, Zhao M, Bai Y, et al. Retinal ischemia/reperfusion injury is mediated by Toll-like receptor 4 activation of NLRP3 inflammasomes. Invest Ophthalmol Vis Sci. 2014;55:5466-75.

51. Dostert C, Guarda G, Romero JF, et al. Malarial hemozoin is a Nalp3 inflammasome activating danger signal. PLoS One. 2009;4: e6510.

52. He X, Mekasha S, Mavrogiorgos N, et al. Inflammation and fibrosis during Chlamydia pneumoniae infection is regulated by IL-1 and the NLRP3/ASC inflammasome. J Immunol. 2010;184:5743-54.

53. Ge J, Tian J, Yang H, et al. Alpha7 nicotine acetylcholine receptor agonist PNU-282987 attenuates acute lung injury in a cardiopulmonary bypass model in rats. Shock. 2017;47:474-9.

54. Hughes FM Jr., Hill HM, Wood CM, et al. The NLRP3 inflammasome mediates inflammation produced by bladder outlet obstruction. J Urol. 2016;195:1598-605.

55. Zhou YH, Han QF, Wang LH, et al. High mobility group box 1 protein attenuates myocardial ischemia reperfusion injury via inhibition of the p38 mitogen-activated protein kinase signaling pathway. Exp Ther Med. 2017;14:1582-8.

56. Cai X, Wang X, Li J, et al. Protective effect of glycyrrhizin on myocardial ischemia/reperfusion injury-induced oxidative stress, inducible nitric oxide synthase and inflammatory reactions 
through high-mobility group box 1 and mitogen-activated protein kinase expression. Exp Ther Med. 2017;14:1219-26.

57. Chen CB, Liu LS, Zhou J, et al. Up-regulation of HMGB1 exacerbates renal ischemia-reperfusion injury by stimulating inflammatory and immune responses through the TLR4 signaling pathway in mice. Cell Physiol Biochem. 2017;41:2447-60.

58. Hori O, Brett J, Slattery T, et al. The receptor for advanced glycation end products (RAGE) is a cellular binding site for amphoterin. Mediation of neurite outgrowth and co-expression of rage and amphoterin in the developing nervous system. J Biol Chem. 1995;270:25752-61.

59. Park JS, Svetkauskaite D, He Q, et al. Involvement of toll-like receptors 2 and 4 in cellular activation by high mobility group box 1 protein. J Biol Chem. 2004;279:7370-7.

60. Van Opdenbosch N, Van Gorp H, Verdonckt M, et al. Caspase-1 engagement and TLR-induced c-FLIP expression suppress
ASC/caspase-8-dependent apoptosis by inflammasome sensors NLRP1b and NLRC4. Cell Rep. 2017;21:3427-44.

61. Lamkanfi M, Sarkar A, Vande Walle L, et al. Inflammasomedependent release of the alarmin HMGB1 in endotoxemia. J Immunol. 2010;185:4385-92.

62. Van Opdenbosch N, Gurung P, Vande Walle L, et al. Activation of the NLRP1b inflammasome independently of ASC-mediated caspase1 autoproteolysis and speck formation. Nat Commun. 2014;5:3209.

63. Geng Y, Ma Q, Liu YN, et al. Heatstroke induces liver injury via IL1 beta and HMGB1-induced pyroptosis. J Hepatol. 2015;63:622-33.

64. Yang J, Zhao Y, Zhang P, et al. Hemorrhagic shock primes for lung vascular endothelial cell pyroptosis: role in pulmonary inflammation following LPS. Cell Death Dis. 2016;7:e2363.

65. Kuipers MT, Aslami H, Janczy JR, et al. Ventilator-induced lung injury is mediated by the NLRP3 inflammasome. Anesthesiology. 2012;116:1104-15. 